\title{
Homotopy Perturbation Method for MHD Boundary Layer Flow with Low Pressure Gradient over a Flat Plate
}

\author{
A. K. Jhankal \\ Department of Applied Mathematics, Birla Institute of Technology, Mesra, Ranchi, Extension Center-Jaipur, \\ Jaipur-302 017, India \\ Email: anujjhankal@yahoo.com
}

\begin{abstract}
Using He's Homotopy Perturbation Method (HPM), the system of non-linear partial differential equations governing the MHD boundary layer equations with low pressure gradient over a flat plate are solved. The main advantage of HPM is that it does not require the small parameters in the equations and hence the limitations of traditional perturbation can be eliminated. The influence of various relevant physical characteristics are presented and discussed.
\end{abstract}

Keywords: MHD, Boundary layer flow, Homotopy perturbation method (HPM), Pressure gradient.

\section{NOMENCLATURE}

$\begin{array}{ll}B_{0} & \text { constant applied magnetic field } \\ f & \text { electric field } \\ \text { Pr } & \text { prandtl number } \\ u & \text { velocity component in } \mathrm{x} \text { direction } \\ U_{\infty} & \text { free stream velocity } \\ v & \text { velocity component in y direction } \\ x & \text { coordinate in direction of surface motion } \\ y & \text { coordinate in direction normal to surface ce } \\ & \text { motion } \\ M & \text { dimensionless magnetic field parameter } \\ & \text { absolute temperature }\end{array}$

$p \quad$ pressure

\section{Greek symbols}

$\eta \quad$ dimensionless similarity variable

$\rho \quad$ density of fluid

$v \quad$ kinematic viscosity

$\Psi \quad$ stream function

$\alpha \quad$ thermal diffusivity

$\theta$ dimensionless temperature

$\lambda \quad$ pressure gradient parameter

$\sigma \quad$ electrical conductivity

\section{Superscript}

derivative with respect to $\eta$ 
A. K. Jhankal/ JAFM, Vol. 7, No. 1, pp. 177-185, 2014.

\section{Subscripts \\ w properties at the plate \\ $\infty \quad$ free stream condition}

\section{INTRODUCTION}

Study of magnetohydrodynamics (MHD) flow with heat and mass transfer play an important role in various industrial applications. Some important applications are cooling of nuclear reactors, liquid metals fluid, power generation system and aero dynamics. It is of importance in connection with many engineering problem, such as sustained plasma confinement for controlled thermo nuclear fusion and electromagnetic casting of metals. MHD finds applications in electromagnetic pumps, crystal growing, MHD coules and bearings, plasma jets and chemical synthesis.

Most of the problem in MHD area are nonlinear. Except a limited number of these problem have precise analytical solution, most of them do not have exact solution, and so these nonlinear equations should be solved using other proper methods. Most scientists believe that the combination of numerical and semi-exact analytical methods can lead to applicable results. In this paper one of the semi-exact method which is called HPM has been introduced and applied in MHD boundary layer flow with low pressure gradient over a flat plat. The initial work in HPM was studied by J. H. He (1998, 2000, 2001, 2005,2009 ) and after that these investigations inspired a lot of researchers Ariel et al. (2006), Beléndez et al. (2008), Bird et al. (2007), Ganji and Ganji (2008), Ganji and Rajabi (2006), Ganji and Sadighi (2006), Hosein et al. (2008), Ma et al. (2008), Siddiqui et al. (2008), Zhang et al. (2008) and Zhang and $\mathrm{He}$ (2006) to solve nonlinear equations with this method.

The present paper deals with the steady MHD laminar forced convection with pith homotopy perturbation method (HPM). The influence of various relevant physical characteristics are presented are discussed.

Basic idea of homotopy perturbation method (HPM): To illustrate the basic ideas of the HPM, we consider the following nonlinear differential equation.
$A(u)-f(r)=0, r \in \Omega$

Subject to the boundary conditions

$B\left(u, \frac{\partial u}{\partial \eta}\right)=0, r \in \Gamma$

Where $\mathrm{A}$ is a general differential operator, $\mathrm{B}$ is a boundary operator, $\mathrm{f}(\mathrm{r})$ is a known analytical function and $\Gamma$ is the boundary of the domain $\Omega$. A can be divided into two parts which are $\mathrm{L}$ and $\mathrm{N}$, where $\mathrm{L}$ is linear and $\mathrm{N}$ is nonlinear. Therefore Eq. 1 can be rewritten as follows:

$\mathrm{L}(u)+N(u)-f(r)=0, r \in \Omega$

By the homotopy perturbation technique, we construct a homotopy

$v(r, p): \Omega \times[0,1] \rightarrow R$, which satisfies:

$H(v, p)=(1-p)\left[L(v)-L\left(u_{0}\right)\right]+p[A(v)-$

$f(r)]=0, p \in[0,1], r \in \Omega$

Where $p \in[0,1]$ is an embedding parameter and $u_{0}$ is an initial approximation that satisfies the boundary condition. Obviously, from these definitions we will have:

$$
\begin{aligned}
& H(v, 0)=L(v)-L\left(u_{0}\right)=0 \\
& H(v, 1)=A(v)-f(r)=0
\end{aligned}
$$

The changing process of $\mathrm{p}$ from zero to one is just that of $v(r, p)$ from $u_{0}(r)$ to $u(r)$. In topology, this is called deformation and $L(v)-L\left(u_{0}\right)$ and $A(v)-$ $f(r)$ are called homotopy. According to the HPM, we can first use the embedding parameter $p$ as a "small parameter" and assuming that the solution of (4) can be written as a power series in $p$ :

$v=v_{0}+p v_{1}+p^{2} v_{2} \ldots$

Setting $p=1$, results in the approximate solution of (1):

$u=\lim _{p \rightarrow 1} v=v_{0}+v_{1}+v_{2}+\cdots$

The convergence and stability of this method was shown by J. H. He (2005).

\section{MATHEMATICAL FORMULATION}

Consider steady flow, with constant free stream velocity $U_{\infty}$ without turbulence over a semi infinite flat plate aligned with the flow. All fluid properties are considered to the constant, in the presence of a transverse magnetic field of uniform strength $B_{0}$ fixed to the plate.

We consider the case of a short circuit problem in which the applied electric field $E=0$, and also assure that the induced magnetic field is small compared to the external magnetic field $B_{0}$. This 
implies a small magnetic Reynolds number, the Joule heating in the energy equation is neglected. The governing boundary layer equations are [Bird et al. (2007)]:

$\frac{\partial u}{\partial x}+\frac{\partial u}{\partial y}=0$

$u \frac{\partial u}{\partial x}+v \frac{\partial u}{\partial y}=-\frac{1}{\rho} \frac{\partial p}{\partial x}+v \frac{\partial^{2} u}{\partial x^{2}}-\frac{\sigma B_{0}^{2}}{\rho} u$

$u \frac{\partial T}{\partial x}+v \frac{\partial T}{\partial y}=\alpha \frac{\partial^{2} T}{\partial y^{2}}$

Along with the boundary conditions for the problem are given by:

$u(x, 0)=0, v(x, 0)=0, T(x, 0)=0, u(x, \infty) \rightarrow U_{\infty}$, $T(x, \infty) \rightarrow T_{\infty}$

The continuity Eq.7 is satisfied by introducing a stream function $\Psi$ such that $u=\frac{\partial \Psi}{\partial y}$ and $=-\frac{\partial \Psi}{\partial x}$

The momentum and energy equations can be transformed into the corresponding ordinary nonlinear differential equations by the following transformation: $\quad \quad \quad=y \sqrt{\frac{U_{\infty}}{v x}}, \Psi=\sqrt{v x U_{\infty}} f(\eta)$,

$\theta(\eta)=\frac{T-T_{\infty}}{T_{w}-T_{\infty}}$

Where $\eta$ is the independent similarity variable. The transformed nonlinear ordinary equations are

$f^{\prime \prime \prime}+\frac{1}{2} f f^{\prime \prime}-M^{2} f^{\prime}=-\lambda$

$\theta^{\prime \prime}+\frac{P r}{2} f f^{\prime}=0$

The transformed boundary conditions are:

$$
f(0)=0, f^{\prime}(0)=0, \theta(0)=1,
$$

$f^{\prime}(\infty) \rightarrow 1, \theta(\infty) \rightarrow 0$.

Where prime denotes differentiation with respect to $\eta, \lambda=-\frac{\mathrm{x}}{\rho \mathrm{U}_{\infty}} \frac{\mathrm{dp}}{\mathrm{dx}}$ is the pressure gradient, $M^{2}=\frac{\sigma B_{0}^{2}}{\rho} \frac{x}{U_{\infty}}$ is the dimensionless magnetic parameter and $\operatorname{Pr}=v / \alpha$ is the Prandtl number.

\subsection{Solution with Homotopy Perturbation Method}

According to the HPM, the homotopy form of Eq. (12) and (13) are constructed as follows:

$$
\begin{aligned}
& (1-p)\left(f^{\prime \prime \prime}-M^{2} f^{\prime}\right)+p\left(f^{\prime \prime \prime}+\frac{1}{2} f f^{\prime \prime}-M^{2} f^{\prime}\right) \\
= & -\lambda \\
(1-p)\left(\theta^{\prime \prime}\right)+p\left(\theta^{\prime \prime}+\frac{P r}{2} f f^{\prime}\right)=0 \quad \ldots(16) &
\end{aligned}
$$

We consider $f$ and $\theta$ as the following:

$f=f_{0}+p f_{1}+p^{2} f_{2} \ldots$

$\theta=\theta_{0}+p \theta_{1}+p^{2} \theta_{2} \ldots$
By substituting Eq. (17) into (15) and (16), and then (I) Terms independent of $p$ give

$f_{0}^{\prime \prime \prime}-M^{2} f_{0}^{\prime}=-\lambda$

$\theta_{0}^{\prime \prime}=0$

The boundary conditions are

$f_{0}(0)=0, f_{0}^{\prime}(0)=0, f_{0}^{\prime}(\infty)=1, \theta_{0}(0)=1$,

$\theta_{0}(\infty)=0$.

(II) Terms containing only $p$ give

$f_{1}^{\prime \prime \prime}-M^{2} f_{1}^{\prime}+\frac{1}{2} f_{0} f_{0}^{\prime \prime}=0$
$\theta_{1}^{\prime \prime}+\frac{P r}{2} f_{0} \theta_{0}=0$

The boundary conditions are

$f_{1}(0)=0, f_{1}^{\prime}(0)=0, f_{1}^{\prime}(\infty)=0, \quad \theta_{1}(0)=0$,

$\theta_{1}(\infty)=0$

(III) Terms containing only $p^{2}$ give

$f_{2}^{\prime \prime \prime}-M^{2} f_{2}^{\prime}+\frac{1}{2}\left(f_{0} f_{1}^{\prime \prime}+f_{1} f_{0}^{\prime \prime}\right)=0$

$\theta_{2}^{\prime \prime}+\frac{P r}{2}\left(f_{0} \theta_{1}^{\prime}+f_{1} \theta_{0}^{\prime}\right)=0$

The boundary conditions are

$f_{2}(0)=0, f_{2}^{\prime}(0)=0, f_{2}^{\prime}(\infty)=0, \theta_{2}(0)=$

$0, \theta_{2}(\infty)=0$

Solving Eq. (18)-(19), (21)-(22) and (24)-(25) with boundary conditions (20), (23) and (26) respectively, we have

$f_{0}=C_{1}+C_{2} e^{m \eta}+C_{3} e^{-m \eta}+\frac{\lambda \eta}{M^{2}}$

$f_{1}=C_{4}+C_{5} e^{m \eta}+C_{6} e^{-m \eta}+A_{3} \eta e^{m \eta}+$

$A_{4} \eta e^{-m \eta}+A_{5} \eta^{2} e^{m \eta}+A_{6} \eta^{2} e^{-m \eta}+A_{7} e^{2 m \eta}+$

$A_{8} e^{-2 m \eta}+A_{9} \eta$

$f_{2}=C_{7}+C_{8} e^{m \eta}+C_{9} e^{-m \eta}+A_{11} \eta e^{m \eta}+$

$A_{12} \eta e^{-m \eta}+A_{13} \eta^{2} e^{m \eta}+A_{14} \eta^{2} e^{-m \eta}+$

$A_{15} \eta^{3} e^{m \eta}+A_{16} \eta^{3} e^{-m \eta}+A_{17} \eta^{4} e^{m \eta}+$

$A_{18} \eta^{4} e^{-m \eta}+A_{19} e^{2 m \eta}+A_{20} e^{-2 m \eta}+A_{21} \eta e^{2 m \eta}+$

$A_{22} \eta e^{-2 m \eta}+A_{23} \eta^{2} e^{2 m \eta}+A_{24} \eta^{2} e^{-2 m \eta}+$

$A_{25} e^{3 m \eta}+A_{26} e^{-3 m \eta}+A_{27} \eta^{3}+A_{28} \eta^{2}+A_{29} \eta$

$\theta_{0}=-\frac{\eta}{6}+1$

$\theta_{1}=-\frac{P r}{2 M^{2}}\left(\frac{1}{3 M}+1\right) C_{2} e^{M \eta}+\frac{P r}{2 M^{2}}\left(\frac{1}{3 M}-\right.$

1) $C_{3} e^{-M \eta}+\frac{C_{2} P r}{12 M^{2}} \eta e^{M \eta}+\frac{C_{3} P r}{2 M^{2}} \eta e^{-M \eta}+\frac{\lambda P r}{144 M^{2}} \eta^{4}-$

$\frac{P r}{12}\left(\frac{\lambda}{M^{2}}-\frac{C_{1}}{6}\right) \eta^{3}-\frac{C_{1} P r}{4} \eta^{2}+B_{1} \eta+B_{2}$

$\theta_{2}=\left(\frac{B_{3}}{M^{2}}-\frac{2 B_{5}}{M^{3}}+\frac{6 B_{7}}{M^{4}}-\frac{24 B_{9}}{M^{5}}\right) e^{M \eta}+\left(\frac{B_{4}}{M^{2}}-\frac{2 B_{6}}{M^{3}}+\right.$

$\left.\frac{6 B_{8}}{M^{4}}+\frac{24 B_{10}}{M^{5}}\right) e^{-M \eta}+\left(\frac{B_{5}}{M^{2}}-\frac{4 B_{5}}{M^{3}}+\frac{18 B_{9}}{M^{4}}\right) \eta e^{M \eta}+$

$\left(\frac{B_{6}}{M^{2}}+\frac{4 B_{8}}{M^{3}}+\frac{18 B_{10}}{M^{4}}\right) \eta e^{-M \eta}+\left(\frac{B_{7}}{M^{2}}-\frac{6 B_{9}}{M^{3}}\right) \eta^{2} e^{M \eta}+$

$\left(\frac{B_{8}}{M^{2}}+\frac{6 B_{10}}{M^{3}}\right) \eta^{2} e^{-M \eta}+\frac{B_{9}}{M^{2}} \eta^{3} e^{M \eta}+\frac{B_{10}}{M^{2}} \eta^{3} e^{-M \eta}+$

$\left(\frac{B_{11}}{4 M^{2}}-\frac{B_{13}}{M^{3}}\right) e^{2 M \eta}++\left(\frac{B_{12}}{4 M^{2}}+\frac{B_{14}}{4 M^{3}}\right) e^{-2 M \eta}+$ 
A. K. Jhankal/ JAFM, Vol. 7, No. 1, pp. 177-185, 2014.

$\frac{B_{13}}{4 M^{2}} \eta e^{2 M \eta}+\frac{B_{14}}{4 M^{2}} \eta e^{-2 M \eta}+\frac{B_{15}}{30} \eta^{6}+\frac{B_{16}}{20} \eta^{5}+$

$\frac{B_{17}}{12} \eta^{4}+\frac{B_{18}}{6} \eta^{3}+\frac{B_{19}}{2} \eta^{2}+B_{20} \eta+B_{21}$

The constant coefficients, can be calculated using boundary conditions, the boundary condition $\eta=\infty$ were replaced by those at $\eta=6$ in accordance with standard practice in the boundary layer analysis. If $\mathrm{p} \rightarrow 1$, we find the approximate solution of Eq. (15) and (16).

The constant coefficients $A_{i}(i=1,2,3, \ldots .29), B_{j}(j=$ $1,2,3, \ldots .21)$ and $C_{k}(k=1,2,3,4,5,6)$ are defined as in the Appendix.

\section{RESULTS AND DISCUSSION}

The numerical results are obtained for low pressure gradient $(\lambda) 0.05$ and 0.1 and fixed value of Prandtl number (Pr) 1.0 and for various values of magnetic parameter $\mathrm{M}$. The effect of magnetic parameter $\mathrm{M}$ on the velocity and temperature are presented in Fig. 1 to 4 .

On the other hand, the skin friction coefficient $f^{\prime \prime}(0)$ and heat transfer coefficient $-\theta^{\prime}(0)$ against magnetic parameter $(\mathrm{M})$, for $\operatorname{Pr}=1.0$ and $\lambda=0.05$ and 0.1 are presented in Fig. 5 and 6 respectively.

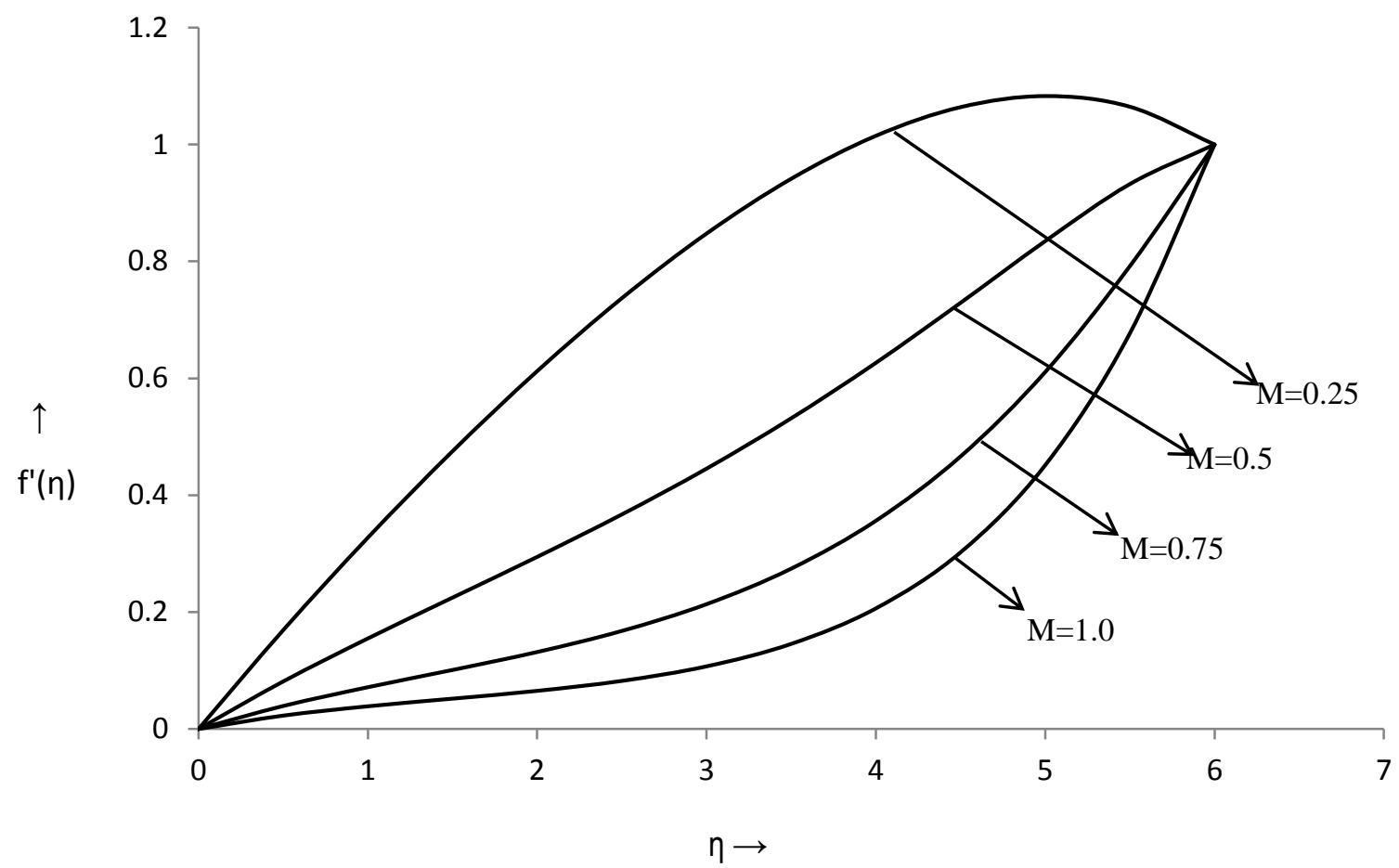

Fig. 1. Velocity profiles f' for various values of magnetic parameter $M$ when $\operatorname{Pr}=1.0$ and $\lambda=0.05$. 
A. K. Jhankal/ JAFM, Vol. 7, No. 1, pp. 177-185, 2014.

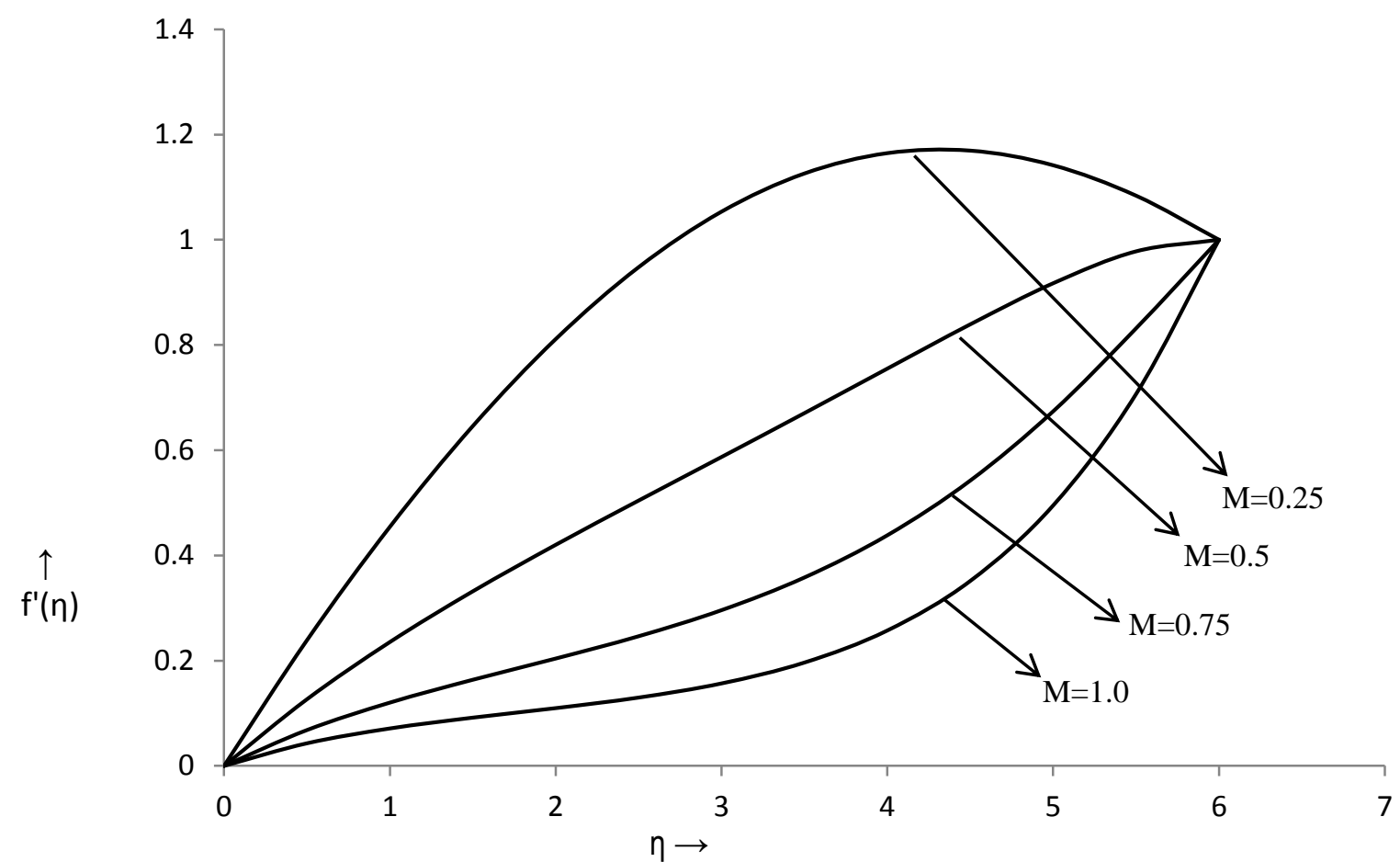

Fig. 2 . Velocity profiles for various values of magnetic parameter $M$ when $\operatorname{Pr}=1.0$ and $\lambda=0.1$.

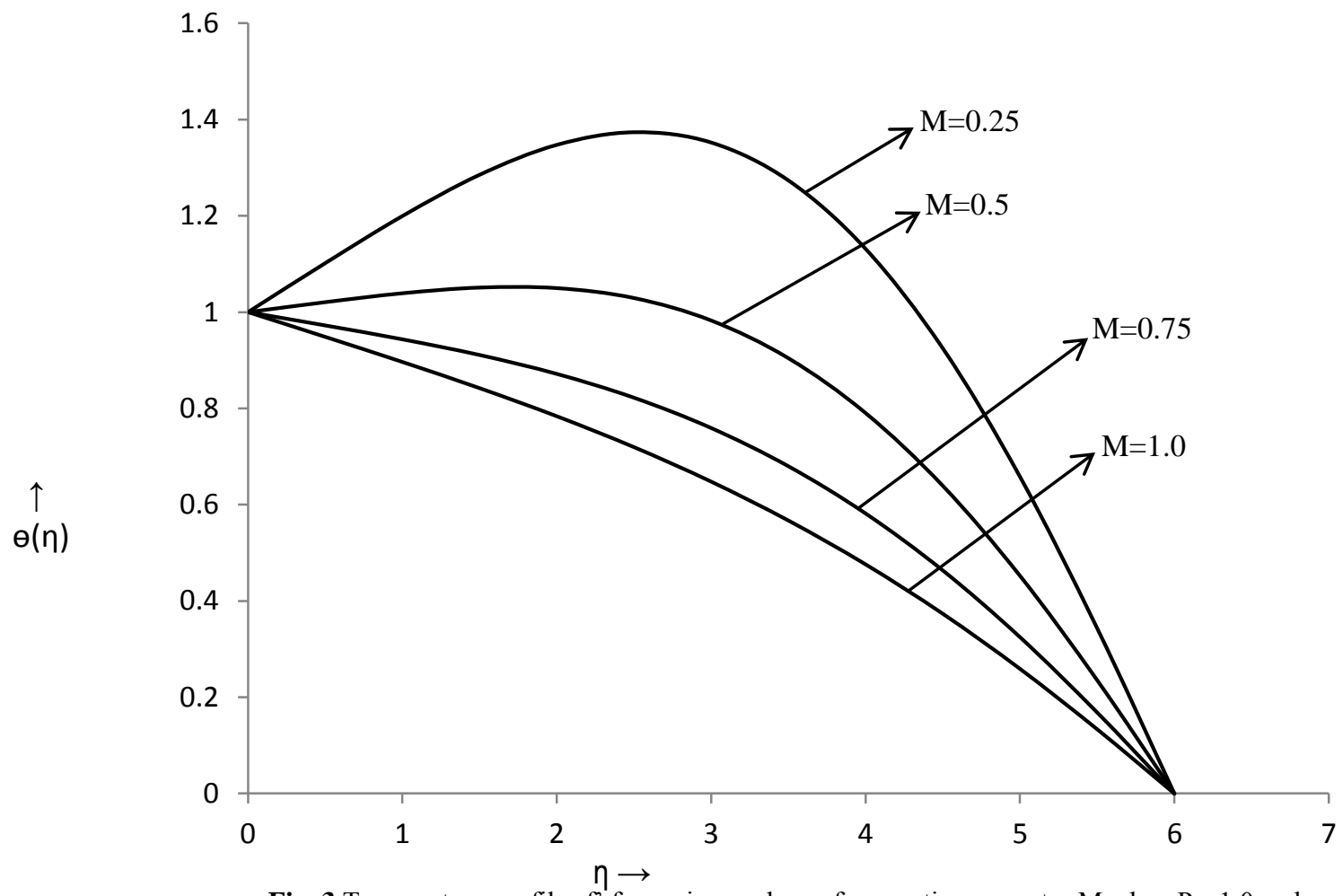

Fig. 3. Temperature profiles $\mathrm{f}^{\prime}$ for various values of magnetic parameter $\mathrm{M}$ when $\mathrm{Pr}=1.0$ and $\lambda=0.05$ 
A. K. Jhankal/ JAFM, Vol. 7, No. 1, pp. 177-185, 2014.

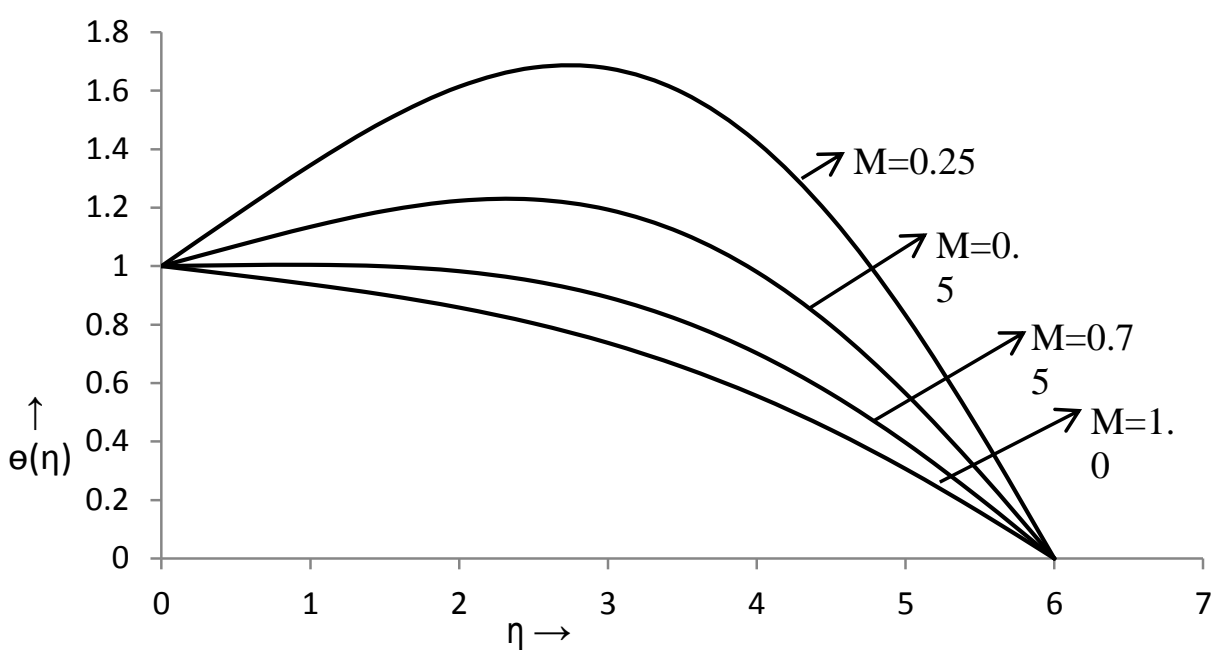

Fig. 4. Temperature profiles $f^{\prime}$ for various values of magnetic parameter $M$ when $\operatorname{Pr}=1 \cap$ and $\lambda=n 1$

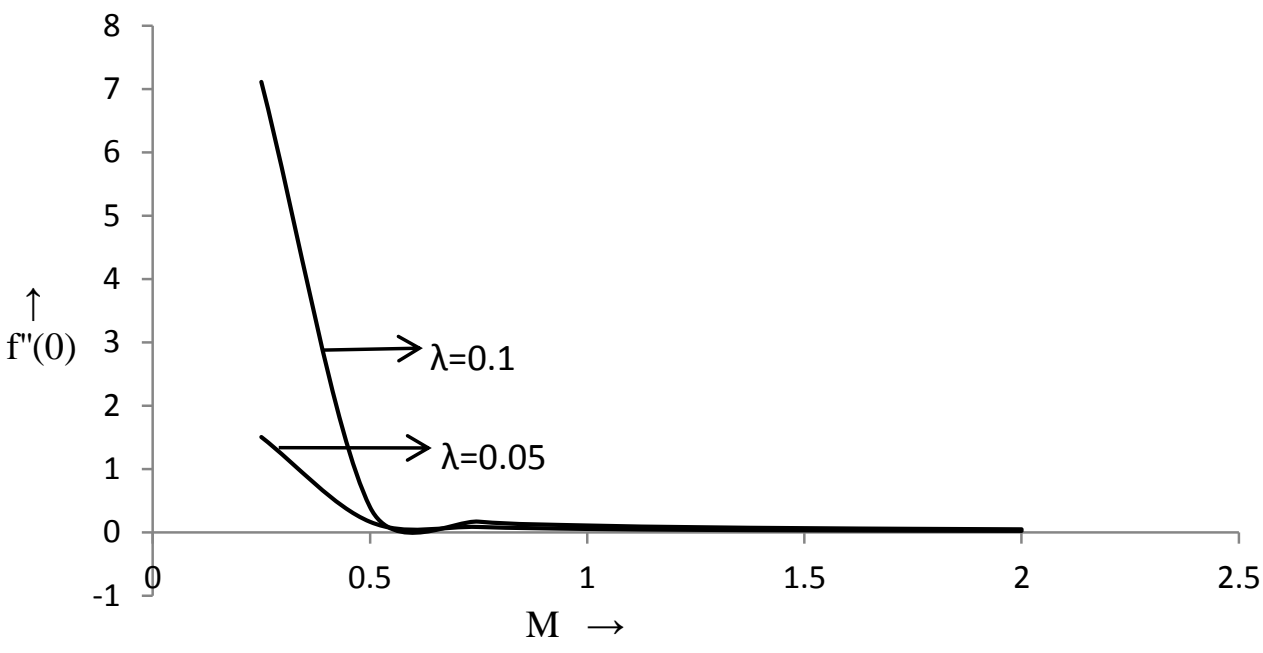

Fig. 5. Cofficient of skin friction against maganatic parmmeter for $\operatorname{Pr}=1.0$

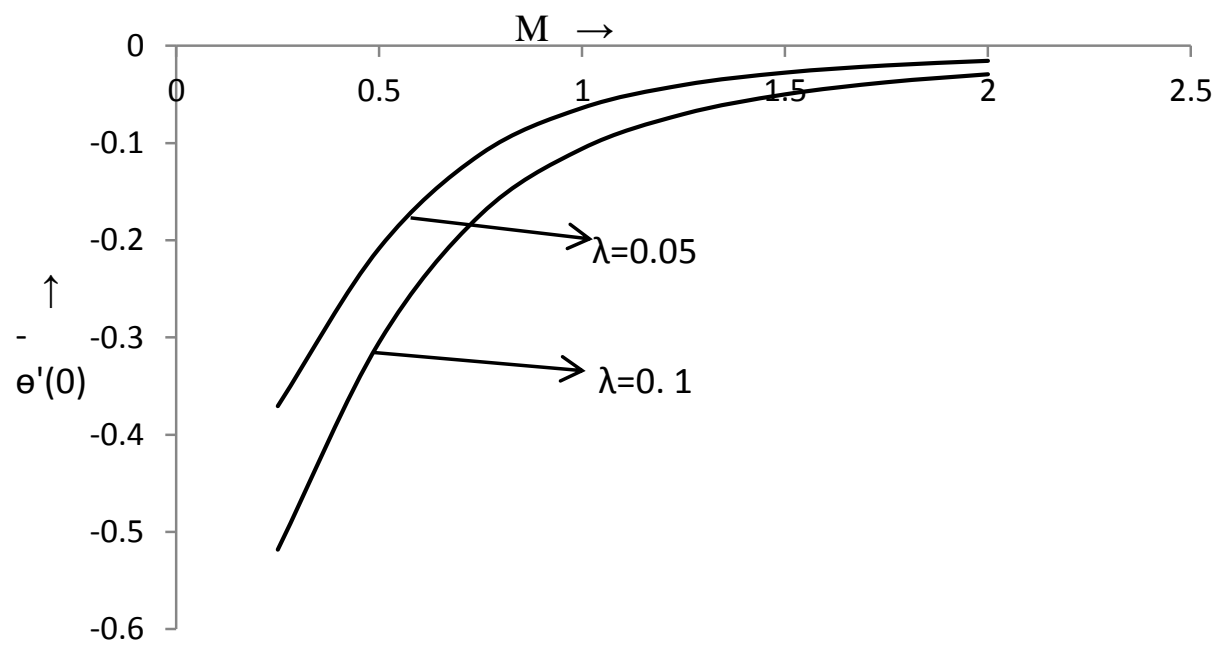

Fig. 6. Nusselt number against maganatic parmmeter for $\operatorname{Pr}=1.0$ 


\section{CONCLUSION}

In this study, the momentum and energy equations, was solved with homotopy perturbation method (HPM) for MHD boundary layer flow with low pressure gradient over a flat plate.

We notice from figures 1 to 4 that for given $\lambda$ the velocity and thermal boundary layer thicknesses decrease with the increasing $M$.

The skin friction and Nusselt number against the magnetic interaction parameter $\mathrm{M}$, for $\mathrm{Pr}=1.0$ are shown in figures 5 and 6 respectively. It is noted that for increasing value of $\lambda$, the skin friction increases but it decreases with the increasing values of $\mathrm{M}$, whereas opposite phenomenon occurs in Nusselt number.

\section{REFERENCES}

Ariel, P.D., Hayat, T. and Asghar, S. (2006). "Homotopy perturbation method and axisymmetric Flow over a stretching sheet", Internat J. Nonlinear Sci. Numer. Simul, 7(4), pp. 399-406.

Beléndez, A., Beléndez, T., Márquez, A. and Neipp, C. (2008). "Application of He's homotopy perturbation method to conservative truly nonlinear oscillators", Chaos, Solitons \& Fractals, 37(3), pp. 770-780.

Bird, R. B., Stewart, W. E. and Lightfoot, E. N. (2007). Transport Phenomena, $2^{\text {nd }}$ ed. John Wiley \& Sons, UK.

Ganji, Z.Z. and Ganji, D. D. (2008).“Approximate Solutions of Thermal Boundary-layer Problems in a Semi-infinite Flat Plate by using He's Homotopy Perturbation Method", International Journal of Nonlinear Sciences and Numerical Simulation, 9(4), pp. 415-422.

Ganji, D. D. and Rajabi, A. (2006).“Assessment of homotopy-perturbation and perturbation methods in heat radiation equations", Internat. Comm. Heat Mass Transfer, 33, pp. 391-400.

Ganji, D. D. and Sadighi, A. (2006).“Application of He's Homotopy-perturbation Method to Nonlinear Coupled Systems of Reaction-diffusion Equations", Int. J. Nonl. Sci. and Num. Simu, 7(4), pp. 411-418.

He, J.H. (2009)."An elementary introduction to the homotopy perturbation method", Computers \& Mathematics with Applications, Volume 57, Issue 3, pp. 410-412.

He, J.H. (1998).“Approximate analytical solution for seepage flow with fractional derivatives in porous media", J. Comput. Math. Appl.Mech. Eng , pp. 167, pp. 57-68.

He, J.H. (2000). "A review on some new recently developed nonlinear analytical techniques", Int J Nonlinear Sci Numer Simul, 1, pp.51-70.
He, J.H. (2001). "Modified Lindstedt-Poincare methods for some non-linear oscillations. Part III: double series expansion", Int. J. Nonlinear Sci Numer Simul, 2, pp. 317-320.

He, J.H. (2005). "Homotopy perturbation method for bifurcation on nonlinear problems", Int. J. Non-linear Sci. Numer. Simul, 6, pp. 207-208.

Hosein Nia, S.H., Ranjbar, A.N., Ganji, D. D., Soltani, H. and Ghasemi, J. (2008)."Maintaining the stability of nonlinear differential equations by the enhancement of HPM", Physics Letters A, 372(16), pp. 2855-2861.

Ma, X., Wei, L. and Guo, Z. (2008)."He's homotopy perturbation method to periodic solutions of nonlinear Jerk equations", Journal of Sound and Vibration, 314, pp. 217-227.

Siddiqui, A.M., Zeb, A., Ghori, Q. K. and Benharbit, A. M. (2008)."Homotopy perturbation method for heat transfer flow of a third grade fluid between parallel plates", Chaos, Solutions\& Fractals, 36(1), pp. 182192.

Zhang, B.G., Li, S.Y. and Liu, Z.R. (2008)."Homotopy perturbation method for modified Camassa-Holm and Degasperis-Procesi", Physics Letters A, 372(11), pp. 1867-1872.

Zhang, L.N. and He, J. H. (2006)."Homotopy perturbation method for the solution of the electrostatic potential differential equation", Mathematical Problems in Engineering, Art. No. 83878.

\section{APPENDIX}

$$
\begin{aligned}
& A_{1}=e^{M \eta}-e^{-M \eta}, \\
& A_{2}=\frac{\lambda}{M^{2}}\left(1-e^{-M \eta}\right), \\
& C_{2}=\frac{1-A_{2}}{M A_{1}} \text {, } \\
& C_{3}=\frac{1-A_{2}}{M A_{1}}+\frac{\lambda}{M^{3}}, \\
& C_{1}=-\left(C_{2}+C_{3}\right) \text {, } \\
& A_{3}=-\frac{C_{1} C_{2}}{4}-\frac{3 \lambda C_{2}}{4 M^{5}} \text {, } \\
& A_{4}=-\frac{C_{1} C_{3}}{4}+\frac{3 \lambda C_{3}}{4 M^{5}}, \\
& A_{5}=\frac{\lambda C_{2}}{4 M^{4}} \\
& A_{6}=\frac{\lambda C_{3}}{4 M^{4}} \text {, } \\
& A_{7}=-\frac{C_{2}^{2}}{12 M} \text {, } \\
& A_{8}=\frac{C_{3}^{2}}{12 M} \text {, } \\
& A_{9}=-\frac{2 C_{2} C_{3}}{M^{2}} \text {, } \\
& A_{10}=\left(A_{3}+6 M A_{3}+12 A_{5}+36 M A_{6}\right) e^{6 M} \\
& +\left(A_{4}-6 M A_{4}+12 A_{6}\right. \\
& \left.-36 M A_{6}\right) e^{-6 M}+2 M A_{7} e^{12 M} \\
& -2 M A_{8} e^{-12 M}+A_{9} \\
& C_{5}=\frac{\left(A_{3}+A_{4}+2 M A_{7}-2 M A_{6}+A_{9}\right) e^{-6 M}}{M A_{1}}-\frac{A_{10}}{M A_{1}},
\end{aligned}
$$




$$
\begin{aligned}
& C_{6}=\left(C_{5}+2 A_{7}-2 A_{8}\right)+\frac{1}{M}\left(A_{3}+A_{4}+A_{9}\right) \text {, } \\
& C_{4}=-\left(C_{5}+C_{6}+A_{7}+A_{8}\right) \text {, } \\
& A_{11}=-\frac{1}{4 M^{2}}\left\{C_{1}\left(M^{2} C_{5}+2 M A_{3}+2 A_{5}\right)\right. \\
& \left.+5 C_{3} M^{2} A_{7}+M^{2} C_{2} C_{4}\right\} \\
& +\frac{3}{8 M^{3}}\left\{C_{1}\left(M^{2} A_{3}+4 M A_{5}\right)\right. \\
& +C_{2} M^{2} A_{9}+\frac{\lambda}{M^{2}}\left(M^{2} C_{5}+2 M A_{3}\right. \\
& \left.\left.+2 A_{5}\right)\right\} \\
& -\frac{7}{8 M^{4}}\left\{C_{1} M^{2} A_{5}+\frac{\lambda}{M^{2}}\left(M^{2} A_{3}\right.\right. \\
& \left.\left.+4 M A_{5}\right)\right\}+\frac{45}{16} \frac{\lambda A_{5}}{M^{5}} \\
& A_{12}=-\frac{1}{4 M^{2}}\left\{C_{1}\left(-M^{2} C_{6}-2 M A_{4}+2 A_{6}\right)\right. \\
& \left.+5 C_{2} M^{2} A_{8}+M^{2} C_{3} C_{4}\right\} \\
& -\frac{3}{8 M^{3}}\left\{C_{1}\left(M^{2} A_{4}-4 M A_{6}\right)\right. \\
& +C_{3} M^{2} A_{9}+\frac{\lambda}{M^{2}}\left(-M^{2} C_{6}-2 M A_{4}\right. \\
& \left.\left.+2 A_{6}\right)\right\} \\
& -\frac{7}{8 M^{4}}\left\{C_{1} M^{2} A_{6}+\frac{\lambda}{M^{2}}\left(M^{2} A_{4}\right.\right. \\
& \left.\left.-4 M A_{6}\right)\right\}-\frac{45}{16} \frac{\lambda A_{6}}{M^{5}} \\
& A_{13}=-\frac{1}{8 M^{2}}\left\{C_{1}\left(M^{2} A_{3}+4 M A_{5}\right)+C_{2} M^{2} A_{9}\right. \\
& \left.+\frac{\lambda}{M^{2}}\left(M^{2} C_{5}+2 M A_{3}+2 A_{5}\right)\right\} \\
& +\frac{3}{8 M^{3}}\left\{C_{1} M^{2} A_{5}++\frac{\lambda}{M^{2}}\left(M^{2} A_{3}\right.\right. \\
& \left.\left.+4 M A_{5}\right)\right\}-\frac{21}{16} \frac{\lambda A_{5}}{M^{4}} \\
& A_{14}=-\frac{1}{8 M^{2}}\left\{C_{1}\left(M^{2} A_{4}-4 M A_{6}\right)+C_{3} M^{2} A_{9}+\right. \\
& \left.\frac{\lambda}{M^{2}}\left(-M^{2} C_{6}-2 M A_{4}+2 A_{6}\right)\right\}-\frac{3}{8 M^{3}}\left\{C_{1} M^{2} A_{6}+\right. \\
& \left.+\frac{\lambda}{M^{2}}\left(M^{2} A_{4}-4 M A_{6}\right)\right\}-\frac{21}{16} \frac{\lambda A_{6}}{M^{4}} \\
& A_{15}=-\frac{1}{12 M^{2}}\left\{M^{2} A_{5} C_{1}+\frac{\lambda}{M^{2}}\left(M^{2} A_{3}+4 M A_{5}\right)\right\}+ \\
& \frac{3}{8} \frac{\lambda A_{5}}{M^{3}} \\
& A_{16}=-\frac{1}{12 M^{2}}\left\{M^{2} A_{6} C_{1}+\frac{\lambda}{M^{2}}\left(M^{2} A_{4}-4 M A_{6}\right)\right\}- \\
& \frac{3}{8} \frac{\lambda A_{6}}{M^{3}} \\
& A_{17}=-\frac{1}{16} \frac{\lambda A_{5}}{M^{2}}, \\
& A_{18}=-\frac{1}{16} \frac{\lambda A_{6}}{M^{2}} \\
& A_{19}=-\frac{1}{12 M^{3}}\left\{C_{2} C_{5} M^{2}+4 C_{1} A_{7} M^{2}+C_{2}\left(M^{2} C_{5}+\right.\right. \\
& \left.\left.2 M A_{3}+2 A_{5}\right)\right\}+\frac{11}{72 M^{4}}\left\{C_{2} M^{2} A_{3}++C_{2}\left(M^{2} A_{3}+\right.\right. \\
& \left.\left.4 M A_{5}\right)+4 \lambda A_{7}\right\}-\frac{85}{108} \frac{C_{2} A_{5}}{M^{3}}
\end{aligned}
$$

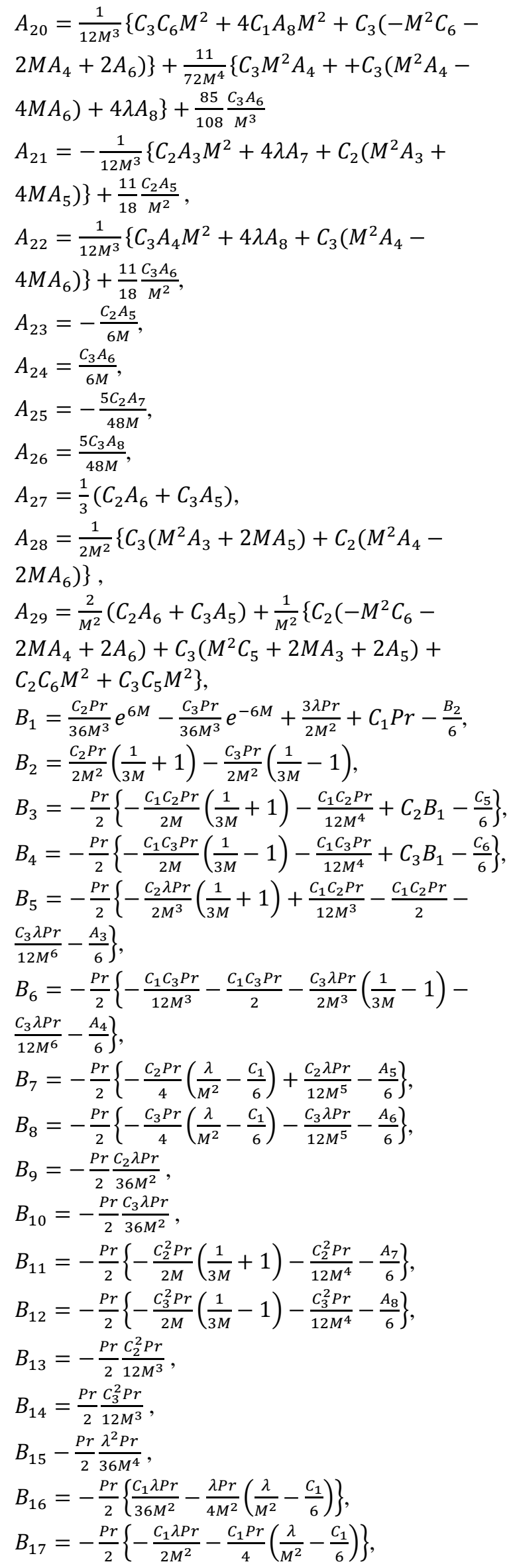




$$
\begin{aligned}
& B_{18}=-\frac{P r}{2}\left\{-\frac{C_{1}^{2} P r}{2}+\frac{\lambda B_{1}}{M^{2}}-\frac{A_{9}}{6}\right\}, \\
& B_{19}=-\frac{P r}{2}\left\{-\frac{C_{2} C_{3} P r}{2 M}\left(\frac{1}{3 M}-1\right)-\frac{C_{2} C_{3} P r}{6 M^{4}}+C_{1} B_{1}-\right. \\
& \left.\frac{C_{2} C_{3} P r}{2 M}\left(\frac{1}{3 M}+1\right)-\frac{C_{4}}{6}\right\}, \\
& B_{20}=\frac{1}{6}\left(-\frac{B_{3}}{M^{2}}+\frac{2 B_{5}}{M^{3}}-\frac{6 B_{7}}{M^{4}}+\frac{24 B_{9}}{M^{5}}-\frac{6 B_{5}}{M^{2}}+\frac{24 B_{7}}{M^{3}}-\right. \\
& \left.\frac{108 B_{9}}{M^{4}}-\frac{36 B_{7}}{M^{2}}-\frac{216 B_{9}}{M^{3}}-\frac{216 B_{9}}{M^{2}}\right) e^{6 M}+\frac{1}{6}\left(-\frac{B_{4}}{M^{2}}+\frac{2 B_{6}}{M^{3}}-\right. \\
& \frac{6 B_{8}}{M^{4}}-\frac{24 B_{10}}{M^{5}}-\frac{6 B_{5}}{M^{2}}-\frac{24 B_{8}}{M^{3}}-\frac{108 B_{10}}{M^{4}}-\frac{36 B_{8}}{M^{2}}-\frac{216 B_{10}}{M^{3}}- \\
& \left.\frac{216 B_{10}}{M^{2}}\right) e^{-6 M}+\frac{1}{6}\left(-\frac{B_{11}}{4 M^{2}}+\frac{B_{13}}{4 M^{3}}-\frac{3 B_{13}}{2 M^{2}}\right) e^{12 M}+ \\
& \left(-\frac{B_{12}}{4 M^{2}}-\frac{B_{14}}{4 M^{3}}-\frac{3 B_{14}}{2 M^{2}}\right) e^{-12 M}-\frac{7776}{5} B_{15}- \\
& \frac{1944}{5} B_{16}-108 B_{17}-136 B_{18}--18 B_{19}-B_{21}, \\
& B_{21}=-\frac{1}{M^{2}}\left(B_{3}+B_{4}+\frac{B_{11}}{4}+\frac{B_{12}}{4}\right) \\
& \quad+\frac{1}{M^{3}}\left(2 B_{5}+2 B_{6}+\frac{B_{13}}{4}-\frac{B_{14}}{4}\right) \\
& \quad-\frac{6}{M^{4}}\left(B_{7}+B_{8}\right)+\frac{24}{M^{5}}\left(B_{9}-B_{10}\right)
\end{aligned}
$$

